\title{
Surgical Management of Sigmoid Volvulus: A Multicenter Observational Study
}

\author{
Keunchul Lee ${ }^{1}$, Heung-Kwon $\mathrm{Oh}^{1}$, Jung Rae Cho ${ }^{1}$, Minhyun Kim ${ }^{1}$, Duck-Woo Kim ${ }^{1}$, Sung-Bum Kang ${ }^{1}$, \\ Hyung-Jin $\mathrm{Kim}^{2}$, Hyoung-Chul Park ${ }^{3}$, Rumi Shin ${ }^{4}$, Seung Chul Heo ${ }^{4}$, Seung-Bum Ryoo ${ }^{5}$, Kyu Joo Park; \\ Seoul Colorectal Research Group (SECOG) \\ ${ }^{1}$ Department of Surgery, Seoul National University Bundang Hospital, Seoul National University College of Medicine, Seongnam; ${ }^{2}$ Department \\ of Surgery, St. Vincent Hospital, College of Medicine, The Catholic University of Korea, Suwon; ${ }^{3}$ Department of Colorectal Surgery, Hallym \\ University Sacred Heart Hospital, Hallym University College of Medicine, Anyang; ${ }^{4}$ Department of Surgery, Seoul Metropolitan Government- \\ Seoul National University Boramae Medical Center, Seoul National University College of Medicine, Seoul; ${ }^{5}$ Department of Surgery, Seoul \\ National University Hospital, Seoul National University College of Medicine, Seoul, Korea
}

Purpose: This study aimed to evaluate real-world clinical outcomes from surgically treated patients for sigmoid volvulus. Methods: Five tertiary centers participated in this retrospective study with data collected from October 2003 through September 2018, including demographic information, preoperative clinical data, and information on laparoscopic/open and elective/emergency procedures. Outcome measurements included operation time, postoperative hospitalization, and postoperative morbidity.

Results: Among 74 patients, sigmoidectomy was the most common procedure $(\mathrm{n}=46)$, followed by Hartmann's procedure $(n=23)$, and subtotal colectomy $(n=5)$. Emergency surgery was performed in 35 cases $(47.3 \%)$. Of the 35 emergency patients, 34 cases $(97.1 \%)$ underwent open surgery, and a stoma was established for 26 patients (74.3\%). Elective surgery was performed in 39 cases (52.7\%), including 21 open procedures (53.8\%), and 18 laparoscopic surgeries (46.2\%). Median laparoscopic operation time was 180 minutes, while median open surgery time was 130 minutes $(\mathrm{P}<0.001)$. Median postoperative hospitalization was 11 days for laparoscopy and 12 days for open surgery. There were 20 postoperative complications (27.0\%), and all were resolved with conservative management. Emergency surgery cases had a higher complication rate than elective surgery cases $(40.0 \%$ vs. $15.4 \%, \mathrm{P}=0.034)$.

Conclusion: Relative to elective surgery, emergency surgery had a higher rate of postoperative complications, open surgery, and stoma formation. As such, elective laparoscopic surgery after successful sigmoidoscopic decompression may be the optimal clinical option.

Keywords: Sigmoid volvulus; Laparoscopy; Elective surgery

Received: November 25, 2019 • Accepted: March 23, 2020

Correspondence to: Heung-Kwon Oh, M.D.

Department of Surgery, Seoul National University Bundang Hospital, 82 Gumi-ro 173beon-gil, Bundang-gu, Seongnam 13620, Korea Tel: +82-31-787-7105, Fax: +82-31-787-4055, E-mail: crsohk@gmail.com ORCID: https://orcid.org/0000-0002-8066-2367

\footnotetext{
- The preliminary results of this study were presented by poster at the annual meeting of the European Society of Coloproctology, held from 28 to 30 September 2016 in Milan, Italy.
}

(C) 2020 The Korean Society of Coloproctology

This is an open-access article distributed under the terms of the Creative Commons Attribution NonCommercial License (https://creativecommons.org/licenses/by-nc/4.0) which permits unrestricted noncommercial use, distribution, and reproduction in any medium, provided the original work is properly cited.

\section{INTRODUCTION}

Colonic volvulus is one of the leading causes of colonic obstruction following colorectal cancer and diverticulitis [1]. More than $95 \%$ of volvulus occurs in the sigmoid colon or cecum [2]. Sigmoid volvulus is a rare disease, but the incidence is relatively high in African, Asian, and Middle Eastern countries where high-fiber diets are consumed [3]. Other reported risk factors include congenital anatomic variation, constipation, neurologic disease, and megacolon [4]. A sigmoid colon with a long mesocolon and narrow mesentery is known to be a susceptibility factor for sigmoid volvulus, and it is more common in males than females [5]. 
Endoscopic detorsion is a useful and common treatment for patients, but the recurrence rate after endoscopic detorsion has historically been near $45 \%$ [6]. Sigmoid volvulus can cause ischemic changes and emergency surgical treatment is required if there are signs of gangrenous intestinal tissue [7]. In the case of emergency surgery, it has been reported that postoperative morbidity is common, and mortality reaches as high as $50 \%$ [8]. It is considered ideal to perform elective surgery directly after resuscitation and endoscopic detorsion, during the same hospital visit $[9,10]$. Nevertheless, the most effective approach to surgical treatment of sigmoid volvulus has long been a matter of controversy.

Minimally invasive surgery has recently gained popularity for several reasons, including shorter hospital stays, less blood loss, and lower analgesic consumption [11]. However, there have been relatively few studies reporting on the optimal surgical treatment of sigmoid volvulus.

In clinical practice, older patients often receive elective surgery instead of emergency surgery because of the high morbidity and mortality rate associated with emergency operations [12]. This study aimed to compare the clinical outcomes between emergency and elective surgery patients treated for sigmoid volvulus.

\section{METHODS}

\section{Patients}

We collected data from all patients who underwent surgery for sigmoid volvulus in 5 tertiary hospitals from October 2003 to September 2018. We collected data on age, sex, body mass index (BMI), American Society of Anesthesiologists (ASA) physical status classification, medical and surgical history, pain attack frequency, surgical methods, morbidity, mortality, length of hospital stay, follow-up period, and recurrence. Emergency surgery was performed in cases of failed endoscopic detorsion during initial treatment, suspicion of infarcted bowel, perforated volvulus, or unstable vital sign. Since the pain attack frequency was various as shown in Table 1 and recurrence was common, it has almost been the indication of elective surgery when the pain attack recurred [6].

This retrospective study was approved by the Institutional Review Board (IRB) of Seoul National University Bundang Hospital (B-1709420112). All participating institutions also had IRB approval to participate in this study before the commencement of data collection and analysis, and the requirement for informed consent was waived.

\section{Surgical procedures}

The method of surgery was determined by the attending surgeon. The procedure was not standardized per a study protocol, but was likely similar among participating hospitals, with some slight technical variabilities across institutions $[13,14]$. The patients were placed in the lithotomy position under general anesthesia. Skin preparation, draping, and patient posture were the same for both open surgery and laparoscopic surgery. Surgical methods
Table 1. Patients characteristics (mean, median, and frequency) stratified by open vs. laparoscopic surgery

\begin{tabular}{|c|c|c|c|}
\hline Variable & $\begin{array}{l}\text { Open } \\
(n=55)\end{array}$ & $\begin{array}{l}\text { Laparoscopic } \\
\quad(n=19)\end{array}$ & P-value \\
\hline Sex & & & 0.491 \\
\hline Female & $8(14.5)$ & $4(21.1)$ & \\
\hline Male & $47(85.5)$ & $15(78.9)$ & \\
\hline Age (yr) & $68.0(9.0-95.0)$ & $70.0(20.0-84.0)$ & 0.432 \\
\hline$B M l^{a}\left(\mathrm{~kg} / \mathrm{m}^{2}\right)$ & $21.1(17.3-27.6)$ & $21.5(19.4-25.2)$ & 0.607 \\
\hline ASA PS classification & & & 0.757 \\
\hline $\mid-\|$ & $37(67.3)$ & $15(78.9)$ & \\
\hline III-IV & $18(32.7)$ & $4(21.1)$ & \\
\hline Previous operation history & & & 0.646 \\
\hline No & $37(67.3)$ & $11(57.9)$ & \\
\hline Yes & $18(32.7)$ & $8(42.1)$ & \\
\hline Comorbidity & & & 0.447 \\
\hline No & $16(29.1)$ & $8(42.1)$ & \\
\hline Yes & 39 (70.9) & $11(57.9)$ & \\
\hline Pain attack frequency ${ }^{b}$ & $2(1-7)$ & $3(1-5)$ & 0.338 \\
\hline
\end{tabular}

Values are presented as number (\%), mean ${ }^{\mathrm{a}}$ (range), or median ${ }^{\mathrm{b}}$ (range).

$\mathrm{BMI}$, body mass index; ASA, American Society of Anesthesiologists; PS, physical status.

were up to the attending surgeon in charge to decide whether to do open or laparoscopic surgery.

For the laparoscopic sigmoidectomy, we performed multiport or reduced-port surgery. The port site was determined by the attending surgeon, with little difference across individuals. A $1.5-\mathrm{cm}$ vertical infra-umbilical incision was made for the camera. Then, 2 to 4 of $5-\mathrm{mm}$ trocars and 3 of $12-\mathrm{mm}$ trocars were inserted into the right lower quadrant, right upper quadrant, left lower quadrant, and left upper quadrant (LUQ) sites as working ports. A pneumoperitoneum was established at a pressure of $12 \mathrm{mmHg}$ by $\mathrm{CO}_{2}$. During the surgery, the patient was placed in the Trendelenburg position. We employed the medial approach for redundant sigmoid colon mobilization. The inferior mesenteric artery was low ligated using a 5-mm Hem-o-lok (Teleflex, Inc., Morrisville, NC, USA). Next, transection of the upper rectum was performed using an endoscopic linear stapler (Echelon 60, Ethicon Inc., Cincinnati, $\mathrm{OH}, \mathrm{USA})$. We then extended the umbilical or LUQ trocar site for delivering the sigmoid colon. The anvil of a circular stapler was inserted into the end of the delivered bowel, and intracorporeal end-to-end anastomosis was performed through the anus (ECS 29, Ethicon Inc.). Open sigmoidectomy was usually performed with a lower midline incision. Most procedures were similar to the laparoscopic procedures, with the exception of the operation sites.

Hartmann's operations performed in this study were all open and emergency surgeries. We made lower midline incisions, the 
same as for open sigmoidectomy, and untwisted the rotated sigmoid colon loop. The procedures for colon mobilization and mesocolon dissection were implemented in a manner similar to that of open sigmoidectomy. After transection of the upper rectum, the proximal stump was pulled out, and an end-colostomy was established.

\section{Statistical analysis}

We analyzed categorical variables as frequencies (percentages) and continuous variables as medians (ranges). We made betweengroups comparisons for the laparoscopic group vs. the open surgery groups using the Mann-Whitney U-test for continuous variables and the chi-square test or Fisher exact test for categorical variables. A P-value of less than 0.05 (2-sided) was regarded as statistically significant. Statistical analyses were performed with $\mathrm{R}$ 3.4.4 (R Core Team, R Foundation for Statistical Computing, Vienna, Austria).

\section{RESULTS}

Patient characteristics are shown in Table 1. A total of 74 patients were enrolled in this study; 62 (83.8\%) males and 12 (16.2\%) females. The patients were divided into open and laparoscopic surgery groups, with 55 patients in the open group ( 8 females) and 19 in the laparoscopic group (4 females). The median ages of the 2 groups were 68 years (range, 9 to 95 years) in the open group and 70 years (range, 20 to 84 years) in the laparoscopic group. There was no statistically significant difference between the groups with respect to BMI, ASA physical status classification, previous operation history, or presence of comorbidities. The patient group was divided into elective surgery and emergency surgery in Table 2; there was no statistical significance except for ASA classification $(\mathrm{P}=0.009)$.

Surgical details for all 74 patients are shown in Fig. 1. Emergency surgery consisted of 21 cases of Hartmann's operation, 5 of total colectomy or subtotal colectomy, and 8 of sigmoidectomy; 19 cases had laparoscopic surgery. In the open group, elective surgery included 19 cases of sigmoidectomy and 2 of Hartmann's operation. Taken together, emergency surgery group had more open surgery $(97.1 \%$ vs. $53.8, \mathrm{P}<0.001)$ and stoma formation $(74.3 \%$ vs. $5.1 \%, \mathrm{P}<0.001)$ than elective surgery group.

Outcomes data for all patients, stratified by emergency or elective surgery, are shown in Table 3. In the elective vs. emergency surgery comparison, there was no difference between the 2 groups with respect to operation time or estimated blood loss (EBL). Median EBL was $200 \mathrm{~mL}$ (range, 75 to $500 \mathrm{~mL}$ ) for emergency surgery, which was more than the $100 \mathrm{~mL}$ (range, 50 to 300 $\mathrm{mL}$ ) observed for elective surgery, although the difference was not statistically significant. The median hospital stay was significantly longer in the emergency group compared to the elective group at 16 days vs. 9 days, respectively $(\mathrm{P}=0.001)$.

A total of 20 cases experienced complications; 6 in the elective
Table 2. Patients characteristics (mean, median, and frequency) stratified by elective vs. emergency surgery

\begin{tabular}{|c|c|c|c|}
\hline Variable & $\begin{array}{l}\text { Elective } \\
(n=39)\end{array}$ & $\begin{array}{l}\text { Emergency } \\
\quad(n=35)\end{array}$ & P-value \\
\hline Sex & & & $>0.999$ \\
\hline Female & $6(15.4)$ & $6(17.1)$ & \\
\hline Male & $33(84.6)$ & $29(82.9)$ & \\
\hline Age (yr) & $62.0(9.0-76.0)$ & $70.0(53.0-95.0)$ & 0.272 \\
\hline $\mathrm{BM}^{\mathrm{a}}\left(\mathrm{kg} / \mathrm{m}^{2}\right)$ & $21.8(17.3-24.5)$ & $20.5(18.5-27.6)$ & 0.120 \\
\hline ASA PS classification & & & 0.009 \\
\hline $1-\|$ & $33(84.6)$ & $19(54.3)$ & \\
\hline III-IV & $6(15.4)$ & $16(45.7)$ & \\
\hline Previous operation history & & & 0.697 \\
\hline No & $24(61.5)$ & $24(68.6)$ & \\
\hline Yes & $15(38.5)$ & $11(31.4)$ & \\
\hline Comorbidity & & & 0.672 \\
\hline No & $14(35.9)$ & $10(28.6)$ & \\
\hline Yes & $25(64.1)$ & $25(71.4)$ & \\
\hline Pain attack frequency ${ }^{b}$ & $2(1-7)$ & $3(1-7)$ & 0.486 \\
\hline Stoma formation & $2(5.1)$ & $26(74.3)$ & $<0.001$ \\
\hline
\end{tabular}

Values are presented as number (\%), mean ${ }^{\mathrm{a}}$ (range), or median (range). BMI, body mass index; ASA, American Society of Anesthesiologists; PS, physical status.

surgery group, and 14 in the emergency surgery group $(\mathrm{P}=0.034)$. The 6 instances of complications in the elective surgery group (out of 39 total elective cases; $15.4 \%$ ) included 1 case of pulmonary complications, 3 of ileus, 1 of wound complications, and 1 of anastomosis site leakage. The 14 instances of complications in the emergency surgery group (out of 35 total emergency cases; $40.0 \%$ ) included 6 cases of pulmonary complications, 3 of ileus, 3 of wound complications, 1 of anastomosis site leakage, and 1 of sepsis. According to the Clavien-Dindo classification, complications were summarized in Table 3.

Postoperative outcomes in the open and laparoscopic surgery groups are shown in Table 4. Median operation time was 180 minutes for the laparoscopic group and 130 minutes for the open group $(\mathrm{P}<0.001)$. There were no statistically significant differences in EBL, or length of hospital stay. Postoperative complications occurred for 18 patients $(32.7 \%)$ in the open group and 2 $(10.5 \%)$ in the laparoscopic group. Specifically, there were 7 cases of pulmonary complications, 4 of ileus, 4 of wound complications, 2 of anastomosis site leakage, and 1 of sepsis in the open surgery group; and 2 cases of ileus in the laparoscopic group. There were no deaths, and all complications responded to conservative care without the need for additional surgery. 


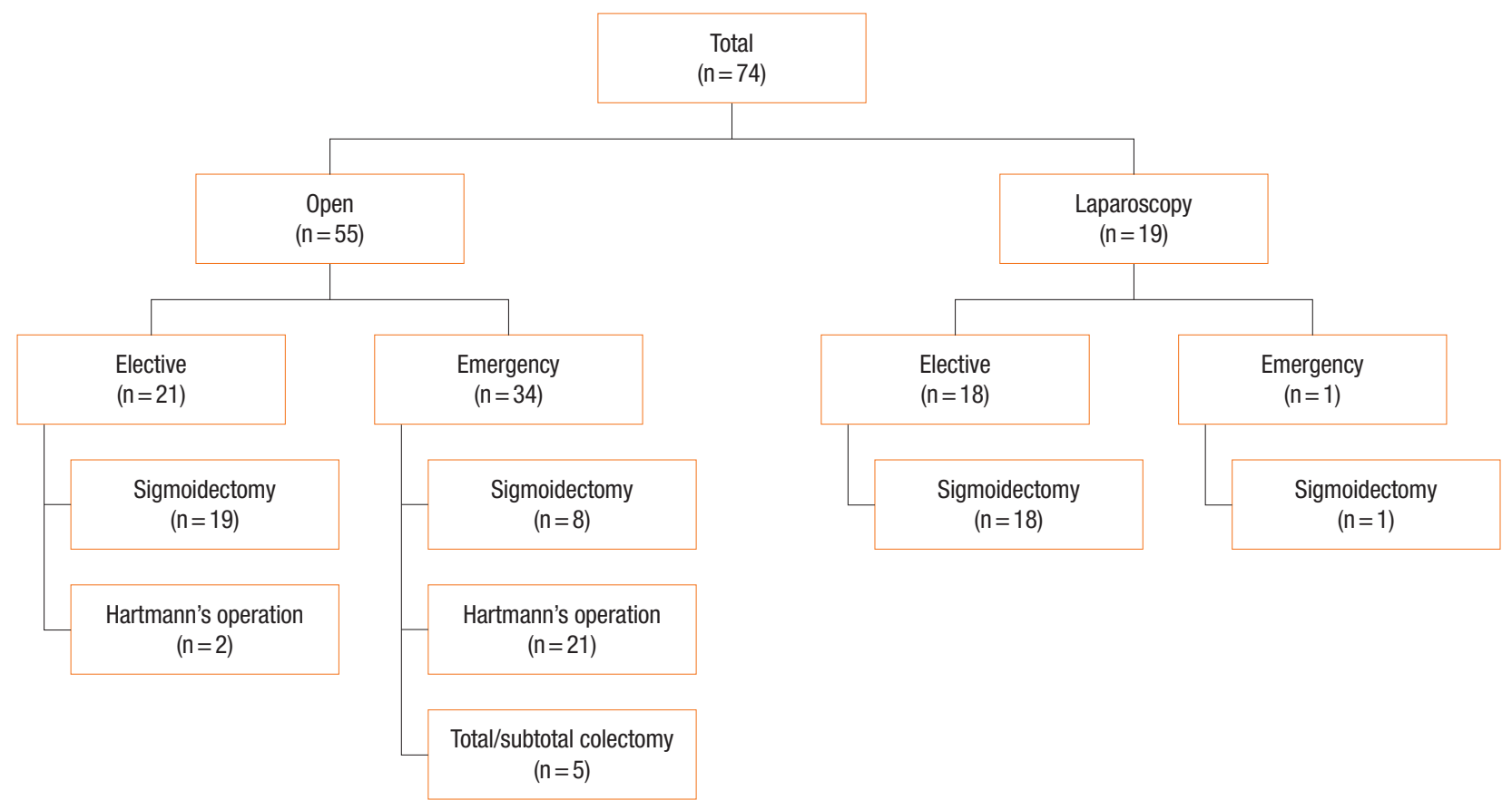

Fig. 1. Sigmoid volvulus operation methods.

Table 3. Outcomes (median, frequency, and range) after elective surgery and emergency surgery

\begin{tabular}{lccc}
\hline Variable & $\begin{array}{c}\text { Elective } \\
(\mathrm{n}=39)\end{array}$ & $\begin{array}{c}\text { Emergency } \\
(\mathrm{n}=35)\end{array}$ & P-value \\
\hline Operation time (min) & $147.5(105.0-180.0)$ & $140.0(108.0-187.5)$ & 0.978 \\
EBL (mL) & $100.0(50.0-300.0)$ & $200.0(75.0-500.0)$ & 0.073 \\
Hospital stay (day) & $9.0(7.0-13.0)$ & $16.0(11.0-23.0)$ & 0.001 \\
Complications & $6(15.4)$ & $14(40.0)$ & 0.034 \\
CDC I & $1(16.7)$ & $3(21.4)$ & \\
CDC II & $4(66.7)$ & $9(64.3)$ & \\
CDC IIla & $1(16.7)$ & $1(7.1)$ & \\
CDC IIIb & $0(0)$ & $0(0)$ & \\
CDC IV & $0(0)$ & $1(7.1)$ & \\
\hline
\end{tabular}

Values are presented as median (range) or number (\%).

EBL, estimated blood loss; CDC, Clavien-Dindo classification.

\section{DISCUSSION}

In this study, we compared postoperative outcomes after laparoscopic and open surgery in patients with sigmoid volvulus overall, and also stratified according to elective or emergency surgery. Elective laparoscopic surgery performed after successful sigmoidoscopic decompression was safe, but emergency surgery had a higher rate of postoperative complications, more open surgery, and more need for stomas compared to elective surgery. In addition, emergency surgery often requires secondary surgery for
Table 4. Outcomes (median, frequency, and range) after open surgery and laparoscopic surgery

\begin{tabular}{lccr}
\hline Variable & $\begin{array}{c}\text { Open } \\
(\mathrm{n}=55)\end{array}$ & $\begin{array}{c}\text { Laparoscopic } \\
(\mathrm{n}=19)\end{array}$ & P-value \\
\hline Operation time (min) & $130.0(98.0-170.0)$ & $180.0(162.5-210.0)$ & $<0.001$ \\
EBL (mL) & $175.0(50.0-500.0)$ & $112.5(50.0-400.0)$ & 0.553 \\
Hospital stay (day) & $12.0(8.0-22.0)$ & $11.0(6.0-13.00)$ & 0.156 \\
Complications & $18(32.7)$ & $2(10.5)$ & 0.114 \\
CDC I & $4(22.2)$ & $0(0)$ & \\
CDC II & $11(61.1)$ & $2(100)$ & \\
CDC IIIa & $2(11.1)$ & $0(0)$ & \\
CDC IIIb & $0(0)$ & $0(0)$ & \\
CDC IV & $1(5.6)$ & $0(0)$ & \\
\hline
\end{tabular}

Values are presented as median (range) or number (\%).

EBL, estimated blood loss; CDC, Clavien-Dindo classification.

stoma repair.

According to a large-scale study conducted in France, the percentage of patients experiencing morbidity was higher, and the length of the hospital stay was longer, for patients undergoing open surgery relative to laparoscopic surgery, regardless of patient age [15]. Similar findings were reported from a multicenter study in the United States, which also estimated cumulative mortality at approximately $10 \%[16]$.

According to Ifversen and Kjaer [17], there is improved longterm survival with surgical management relative to conservative 
management due to high recurrence rates. Colonic resection or percutaneous endoscopic colostomy (PEC) can be performed as a surgical treatment [17]. Frank et al. [18] concluded that PEC may be an alternative management option for recurrent sigmoid volvulus in high-risk patients, but $21 \%$ morbidity and $5 \%$ mortality have been reported. In our study, the morbidity associated with elective open surgery was $15.8 \%$, while the morbidity in the laparoscopic surgery group was only $12.5 \%$.

The levels of morbidity in our study were consistent with previous findings, but the percentage of Hartmann's operations performed in the emergency setting was relatively high. In addition, emergency patients experienced higher rates of stoma formation and surgical complications than elective surgery patients. Among elective surgery patients, the complication rate in the laparoscopic group was lower than that in the open surgery group. Taken together, laparoscopic surgery performed in the elective setting leads to more favorable outcomes than emergency surgery for these high-risk patients, particularly when considering the rates of secondary operations, such as stoma repair.

Laparoscopic surgery is generally known to have better outcomes for oral intake, time to flatus, and length of hospital stay than open surgery [19]. Moreover, the incidence of ileus is known to be less frequent in laparoscopic surgery than open surgery [19]. Hence, since there is little need for radiological imaging for laparoscopic surgery, patients may expect fewer expenses related to postoperative treatment [20]. In our study, in spite of statistical insignificance, laparoscopic surgery also has merits with regards to blood loss, postoperative recovery, and morbidity.

This study has some limitations, including potential bias originating from the retrospective design. Second, there were not many patients with sigmoid volvulus who underwent surgery, despite the multicenter nature of the study. As such, the study was underpowered to detect modest differences in outcomes across groups, such as the open vs. laparoscopic surgery groups. Third, there was no standardized treatment protocol although the treatment procedures are similar. Finally, the follow-up period (median, 31.5 months; range, 1 to 151 months) was not long enough to allow comparisons of long-term outcomes.

In conclusion, emergency surgery had a higher rate of postoperative complications, more open surgery, and more need for stomas compared to elective surgery. Since the typical sigmoid volvulus patient has several comorbidities, emergency surgery, with its higher rate of complications, may be inherently dangerous. Elective laparoscopic surgery performed after successful sigmoidoscopic decompression may be an optimal clinical option.

\section{CONFLICT OF INTEREST}

No potential conflict of interest relevant to this article was reported.

\section{REFERENCES}

1. Ballantyne GH, Brandner MD, Beart RW Jr, Ilstrup DM. Volvulus of the colon: incidence and mortality. Ann Surg 1985;202:83-92.

2. Vogel JD, Feingold DL, Stewart DB, Turner JS, Boutros M, Chun J, et al. Clinical practice guidelines for colon volvulus and acute colonic pseudo-obstruction. Dis Colon Rectum 2016;59:589-600.

3. Kapadia MR. Volvulus of the small bowel and colon. Clin Colon Rectal Surg 2017;30:40-5.

4. Saravia Burgos J, Acosta Canedo A. Megacolon and sigmoid volvulus: incidence and physiopathology. Rev Gastroenterol Peru 2015;35:38-44.

5. Bhatnagar BN, Sharma CL, Gupta SN, Mathur MM, Reddy DC. Study on the anatomical dimensions of the human sigmoid colon. Clin Anat 2004;17:236-43.

6. Ueda M, Onishi T, Hata T, Nishida K, Yanagawa T, Fujita S, et al. Usability of elective laparoscopic sigmoidectomy and feasibility of single-incision laparoscopic surgery for sigmoid volvulus: report of three cases. Int Surg 2015;100:408-13.

7. Larkin JO, Thekiso TB, Waldron R, Barry K, Eustace PW. Recurrent sigmoid volvulus: early resection may obviate later emergency surgery and reduce morbidity and mortality. Ann R Coll Surg Engl 2009;91:205-9.

8. Quénéhervé L, Dagouat C, Le Rhun M, Perez-Cuadrado Robles E, Duchalais E, Bruley des Varannes S, et al. Outcomes of first-line endoscopic management for patients with sigmoid volvulus. Dig Liver Dis 2019;51:386-90.

9. Choi BJ, Jeong WJ, Kim SJ, Lee SC. Single-port laparoscopic surgery for sigmoid volvulus. World J Gastroenterol 2015;21:2381-6.

10. Oren D, Atamanalp SS, Aydinli B, Yildirgan MI, Başoğlu M, Polat $\mathrm{KY}$, et al. An algorithm for the management of sigmoid colon volvulus and the safety of primary resection: experience with 827 cases. Dis Colon Rectum 2007;50:489-97.

11. Allaix ME, Giraudo G, Mistrangelo M, Arezzo A, Morino M. Laparoscopic versus open resection for colon cancer: 10 -year outcomes of a prospective clinical trial. Surg Endosc 2015;29:916-24.

12. Perrot L, Fohlen A, Alves A, Lubrano J. Management of the colonic volvulus in 2016. J Visc Surg 2016;153:183-92.

13. Jeong SY, Park JW, Nam BH, Kim S, Kang SB, Lim SB, et al. Open versus laparoscopic surgery for mid-rectal or low-rectal cancer after neoadjuvant chemoradiotherapy (COREAN trial): survival outcomes of an open-label, non-inferiority, randomised controlled trial. Lancet Oncol 2014;15:767-74.

14. Kang SB, Park JW, Jeong SY, Nam BH, Choi HS, Kim DW, et al. Open versus laparoscopic surgery for mid or low rectal cancer after neoadjuvant chemoradiotherapy (COREAN trial): short-term outcomes of an open-label randomised controlled trial. Lancet Oncol 2010;11:637-45.

15. Alves A, Panis Y, Slim K, Heyd B, Kwiatkowski F, Mantion G, et al. French multicentre prospective observational study of laparoscopic versus open colectomy for sigmoid diverticular disease. $\mathrm{Br}$ J Surg 2005;92:1520-5. 


\section{Coloproctology Keunchul Lee, et al.}

16. Halabi WJ, Jafari MD, Kang CY, Nguyen VQ, Carmichael JC, Mills S, et al. Colonic volvulus in the United States: trends, outcomes, and predictors of mortality. Ann Surg 2014;259:293-301.

17. Ifversen AK, Kjaer DW. More patients should undergo surgery after sigmoid volvulus. World J Gastroenterol 2014;20:18384-9.

18. Frank L, Moran A, Beaton C. Use of percutaneous endoscopic colostomy (PEC) to treat sigmoid volvulus: a systematic review. Endosc Int Open 2016;4:E737-41.
19. Chand M, Siddiqui MR, Rasheed S, Brown G, Tekkis P, Parvaiz A, et al. A systematic review and meta-analysis evaluating the role of laparoscopic surgical resection of transverse colon tumours. Surg Endosc 2014;28:3263-72.

20. Cipriani F, Ratti F, Cardella A, Catena M, Paganelli M, Aldrighetti L. Laparoscopic versus open major hepatectomy: analysis of clinical outcomes and cost effectiveness in a high-volume center. J Gastrointest Surg 2019;23:2163-73. 